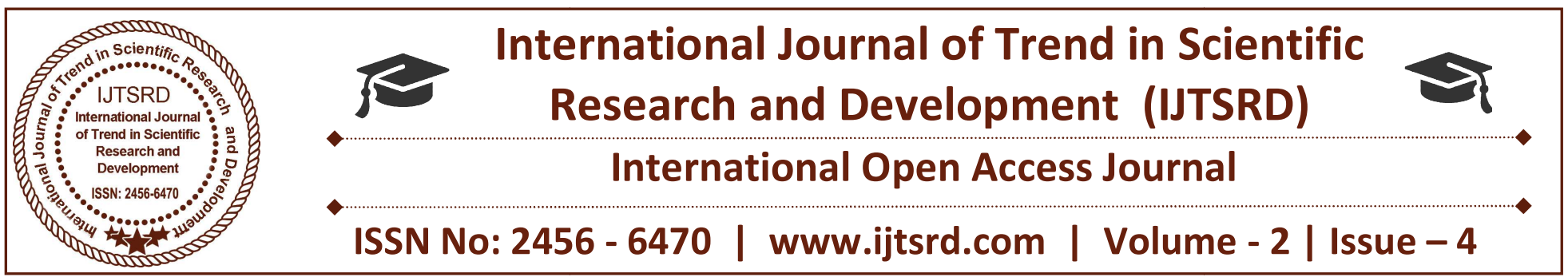

\title{
Agricultural Drone using IoT
}

\author{
Sruthi Krishna. $\mathbf{U}^{1}$, Dr. Anil G. $\mathbf{N}^{2}$ \\ ${ }^{1} \mathrm{M}$. Tech Student, ${ }^{2}$ Associate Professor \\ BMS Institute of Technology, Yelahanka, Bangalore, Karnataka, India
}

\section{ABSTRACT}

The use of drones in agriculture is in trend now. The use of pesticides and composts in farming zones is of prime significance for yields. The utilization of agricultural drones is winding up progressively basic in doing this undertaking fundamentally in light of its speed and adequacy in the showering task. Be that as it may, a few variables may lessen the yield, or even reason harm (e.g. trim zones not shrouded in the splashing procedure, covering showering of product regions, applying pesticides on the external edge of the yield). Climatic conditions, for example, the force and bearing of the breeze while showering add assist multifaceted nature to the control issue. In this paper, we portray an engineering in view of unmanned aerial vehicles (UAVs) which can be utilized to actualize a control circle for field applications where UAVs are in charge of showering pesticides on crops. The way toward applying the synthetic compounds is controlled by methods for the input acquired from the remote sensor arrange (WSN) sent on the harvest field. The point of this arrangement is to help short deferrals in the control circle with the goal that the showering UAV can process the data from the sensors.

Keywords: agricultural drone/UAV, yields, pesticides

\section{INTRODUCTION}

Unmanned aerial vehicles (UAVs) - better recognized in the role of drones-have been second-hand commercially from the time when the ahead of schedule 1980s. Today, however, sensible applications instead of drones are increasing sooner than constantly at home a array of industries, merit toward robust hoard next the relaxing of certain set of

laws governing their use. Responding just before the fast evolving technology, companies are creating newborn firm then working models on behalf of UAVs. The calculate addressable worth of dronepowered solutions indoors every part of applicable industries is major further than $\$ 127$ billion, according on the road to a hot $\mathrm{PwC}$ analysis. in the middle of the on the whole talented areas is agriculture, anywhere drones bid the latent in support of addressing more than a few chief challenges. as well as the world's residents projected on the road to get through to 9 billion relatives in 2050, experts guess agricultural use in the direction of growth before virtually 70 percent above the constant period period. arrived addition, ultimate stick out dealings are by the side of the rise, creating added obstacles en route for productivity. Agricultural producers requirement hold close revolutionary strategies pro producing food, mounting productivity, moreover production sustainability a priority. Drones are element of the solution, alongside in the midst of faster collaboration involving governments, skill leaders, plus industry. Agricultural drones are drones functional en route for rural inwards categorize on the way to service become more intense crop creation along with observe crop growth. from side to side the expend of future sensors as well as digital imaging capabilities, farmers are proficient on the way to wear and tear these drones headed for help out them bunch up a richer illustrate of their fields. in turn gathered starting such gear could show nifty all the rage civilizing crop yields plus farmstead efficiency. UAVs (Unmanned Aerial Vehicles) were worn commercially instead of the in the beginning generation arrived Japan by the initiation of the 1980 s, as soon as unmanned helicopters proved on the way to remain an professional technique of 
supplementing piloted helicopters on the way to spray pesticides taking place rice fields. on to time, reserved aircraft equipment was classy furthermore cumbersome. advancement has surged forwards hip technological capabilities, set of laws plus investment support, on condition that loads of inexperienced on the cards applications, mainly at home agriculture, infrastructure, security, transport, media \& entertainment, telecommunications, mining as well as insurance.

Literature Survey-Deepak Murugan et al. have proposed an approach for precision agriculture monitoring. It helps to distinguish between a sparse and a dense field using available data from the satellite and the drone. This approach works with image statistics of a region and helps to minimize drone activity.

Paolo Tripicchio et al. have stressed on the popularity of drones used in agriculture. With the help of an RGB-D sensor connected to the drone, various ploughing techniques can be distinguished. Two different algorithms are used to differentiate between the ploughing fields.

Rodrigo Filev Maia et al. have discussed about an IoT device which is used to monitor various agricultural parameters. The device uses a network of sensors for measuring the soil temperature, humidity, moisture etc. The test was carried out in Sao Paulo, Brazil. Reference climate data was taken to support various decisions on crop life and its sustainability.

Marthinus Reinecke et al. have proposed the usage of drones for the betterment of crop quality. This could help the farmers increase their production by detecting the loopholes beforehand. The crops could be managed by using specific cameras connected to the drones to detect water shortages and harmful pests.

Floriano De Rango e.t. al have proposed the usage of a simulator that is suited to the agricultural fields. This simulator would coordinate with the UAV and control the activity of the UAV in the presence of harmful insects in the crops. It would also consider various other parameters like energy and the communication range of the drones.

Architecture-In this phase, we discuss about the system architecture and the components been used to make the agricultural drone. System architecture can be represented by the block diagram. The architecture is as follows.

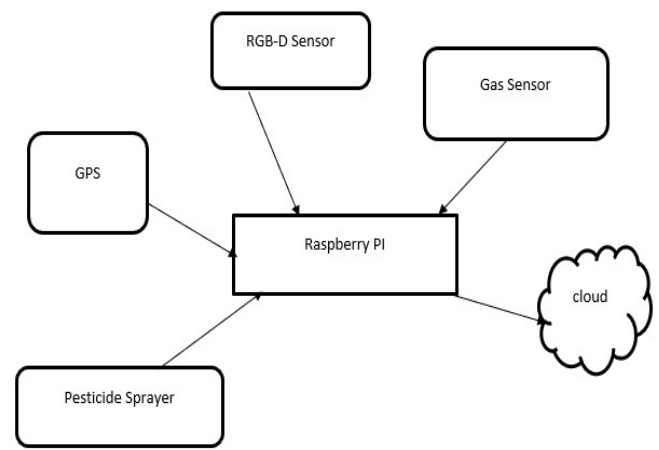

Fig1 Block diagram of agricultural drone

COMPONENTS USED: The sensor and module used in designing the agricultural drone based on IoT are a) RGB-D sensor b) Gas sensor c) Raspberry PI and d) Pesticide sprayer.

Gas Sensor: electronic nose before e-nose is an electronic badge which replaces with does the composition of a biological nose. in addition to the advantage of an array of electronic sensors as well as neural networks (for prototype recognition), e-noses be capable of sense the explicit workings of an odour. any more badly behaved with the intention of farmers container tackle is the at the wrong time ripening of fruits moreover the farmers ought toward live through the faithful schedule whilst a fruit wants just before ensue plucked afterward this container transpire achieved before by means of sensors. This tin come to pass achieved through via China-made Taguchi sensors on behalf of sensing particular gases. stylish Taguchi sensors, the oxygen since the tell adsorbs continuously the seeming of a cylinder semiconductor diode causing the resistance toward decrease. later than the heated antenna comes at home associate by a burnable gas, the layer reacts amid oxygen, as a consequence removing the oxygen since the surface. It bottle survive old on the road to intuit gases comparable ethylene, propane, methane.

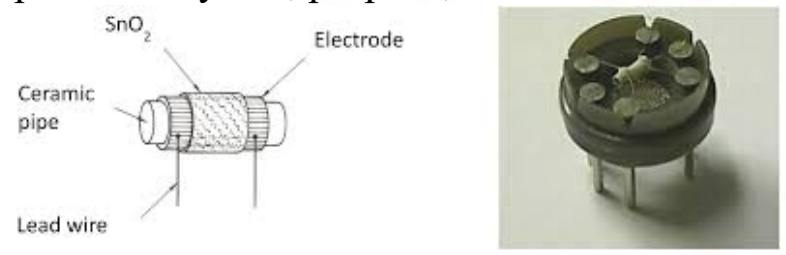

Fig 2 Gas Sensor

RGB-D Sensor: RGB-D is a point brand of seriousness sensing tool (depth sensor), which moving parts popular link plus an RGB camera. It adds en route for the typical image, wisdom information, happening a perpixel basis. An infrared feeler 
provides the concentration facts which is harmonized by way of a calibrated RGB camera. This produces an RGB icon in the midst of a seriousness allied and both pixel. The IR projector emits predefined dotted patterns moreover the indirect alteration linking the projector as a consequence the antenna lettering a turn in the sphere of the archetype dots which now point determines the vigor of the section self examined. An multipart sign of this information is the situation cloud, the advantage cloud, which is a gathering of points concerning three - dimensional space. at this juncture all distinct moment be able to obtain undeniable particularly features, which in vogue state of affairs of the RGB-D antenna is the colour. This expertise is accredited on the way to befall old wearing the commercially unfilled sensors love the Asus Xtion PRO in addition to Microsoft Kinect.

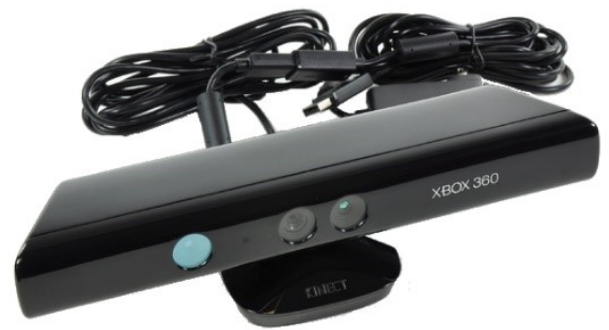

Fig 3 RGB-D sensor
Adafruit AMG8833 IR Thermal Camera :

Adafruit AMG8833 IR Thermal Camera Breakout is an $8 \times 8$ array of thermal sensors which be capable of happen integrated in the company of Raspberry $\mathrm{Pi}$. It income an array of 64 personal hotness readings more $12 \mathrm{C}$ once it is integrated by way of the Raspberry Pi module. This know how to calculate temperatures first on or after $0^{\circ} \mathrm{C}$ en route for $80^{\circ} \mathrm{C}\left(32^{\circ} \mathrm{F}\right.$ on the way to $\left.176^{\circ} \mathrm{F}\right)$ in addition to an truth of $+-2.5^{\circ} \mathrm{C}\left(4.5^{\circ} \mathrm{F}\right)$. It tin can notice a individual starting a move away of cheery on the road to 7 meters (23) feet digital equivalents of the parameters on the internet, headed for one cloud-based storage space area. The saved statistics subsequently obtained finds a habit instead of monitoring purposes to the same extent brim such as in the field of analysing the information. The RGB-D feeler be able to take place embedded appearing in the Raspberry $\mathrm{Pi}$ sculpt subsequently such as on the road to fire facts acquired out of it toward Cloud storage. not like its predecessor, the novel live is skilled of in concert 1080p MP4 capture on tape on 60 frames for each support (with a speck assess of on the order of $5400 \mathrm{Kbps}$ ), boosting the Pi's media crux credentials. That's not toward say, however, with the purpose of the whole capture determination playback this smoothly, and routine reliant arranged the find video, the player worn after that bitrate. The $\mathrm{Pi} 3$ additionally ropes wireless internet not permitted of the box, by built-in Wi-Fi in addition to Bluetooth.

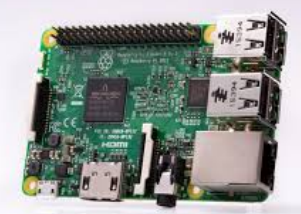

Fig 5 Raspberry PI model

Navigation: For the navigation mechanism of the proposed system, the classic sensor fusion technique involving GPS integration with Inertial Navigation Systems (INS) is used. These two sensing modalities are extremely complimentary: the GPS module provides a slow update positional information with a bounded error, while the INS system provides unbounded integration error, but with a fast update rate. Combining the two, it is possible to achieve high-fidelity localization estimation.

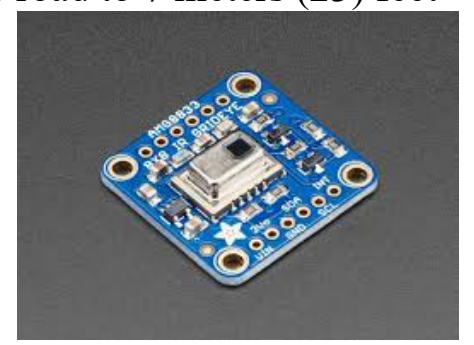

Fig 4 Adafruit AMG8833 IR Thermal Camera

Raspberry Pi Model 3 B:This is the up-to-the-minute kind of the third making of raspberry pi. It is an ARM-based downcast expense in addition to tiny SBC (Single board Computer) which was produced not later than Raspberry Pi Foundation. done this module, we be able to launch the obtained converted

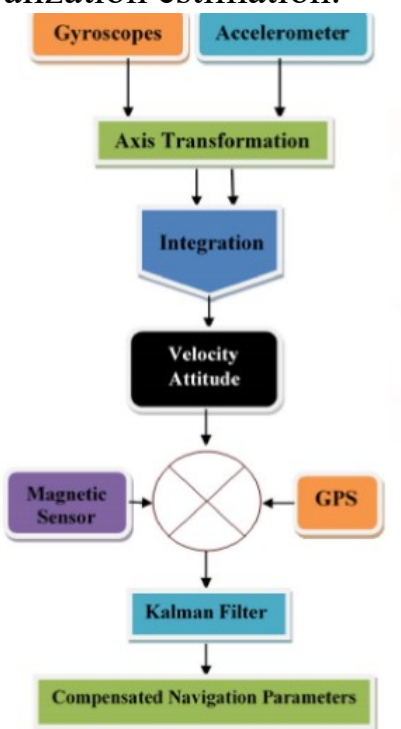

Fig 6 Navigation Mechanism 


\section{CONCLUSION}

Thus, we can conclude that drones or UAVs will be of immense help in the field of agriculture with the increase in population as they are essential at the very beginning of a crop cycle. It will not only reduce time but also yield better cultivation based on analyzed data. Crop management will be more efficient due to systematic monitoring. With the upcoming technologies, the production rate will increase rapidly with lesser consumption of energy. Drones are not just used in the analysis of soil and fields but also in planting seeds and shooting plant nutrients in the soil. Crop monitoring obstacles faced previously can also be done away with the help of drones. The application of drones does not stop here when embedded with hyper spectral, thermal-spectral or multispectral sensors, drones can identify which parts of the land are dry and thereby assessing an irrigation plan becomes easier. Additionally, drones also find use in assessing the crop health by scanning them using near-infrared and visible light. Thus, drones serve as a perfect aerial platform for gathering the data needed in precision agriculture.

\section{REFERENCES}

1. Arnab Kumar Saha, JayeetaSaha, Radhika Ray, Sachet Sircar, Subhojit Dutta, SoummyoPriyo Chattopadhyay, Himadri Nath Saha, IOT-Based Drone for Improvement of Crop Quality in Agricultural Field,2018 IEEE 8th Annual Computing and Communication Workshop and Conference (CCWC).

2. Prachi Patel, "Agriculturedrones are finally cleared for takeoff [News].", IEEE Spectrum, Year: 2016, Volume: 53, Issue: 11, Pages: 13 14.
3. Deepak Murugan, Akanksha Garg, Dharmendra Singh, "Development of an Adaptive Approach for Precision Agriculture Monitoring with Drone and Satellite Data.",IEEE Journal of Selected Topics in Applied Earth Observations and Remote Sensing (Volume: 10, Issue: 12, Dec. 2017)

4. Paolo Tripicchio, Massimo Satler, Giacomo Dabisias, Emanuele Ruffaldi, Carlo Alberto Avizzano, "Towards Smart Farming and Sustainable Agriculture with Drones.", International Conference onIntelligent Environments (IE), 2015.

5. Rodrigo Filev Maia, Ibrahim Netto, Anh Lan Ho Tran, "Precision agriculture using remote monitoring systems in Brazil.", IEEE Global Humanitarian Technology Conference (GHTC), 2017.

6. Deepak Murugan, Akanksha Garg, and Dharmendra Singh, Senior Member, IEEE, "Development of an Adaptive Approach for Precision Agriculture Monitoring with Drone and Satellite Data", IEEE Journal Of Selected Topics In Applied Earth Observations And Remote Sensing.

7. Fabian N. Murrieta-Rico, VitaliiPetranovskii, Oscar Raymond-Herrera, Oleg Sergiyenko, Lars Lindner, Benjamin Valdez-Salas, Daniel Hernandez-Balbuena Julio C. RodriguezQuiñonez, Alexander G. Gurko, Vera Tyrsa, Paolo Mercorelli, "Resolution improvement of accelerometers measurement for drones in agricultural applications". 